\title{
TMT adaptive optics program status report
}

\section{Brent L. Ellerbroek, Sean M. Adkins, David R. Andersen, Jenny Atwood, Arnaud Bastard, et al.}

Brent L. Ellerbroek, Sean M. Adkins, David R. Andersen, Jenny Atwood, Arnaud Bastard, Yong Bo, Marc-Andre Boucher, Corinne Boyer, Peter W. G. Byrnes, Kris Caputa, Shanqiu Chen, Carlos Correia, Raphael Cousty, Joeleff T. Fitzsimmons, Luc Gilles, James Gregory, Glen Herriot, Paul Hickson, Alexis Hill, John Pazder, Hubert Pagès, Thomas Pfrommer, Vladimir A. Reshetov, Scott Roberts, Jean-Christophe Sinquin, Matthias Schoeck, Malcolm Smith, Jean-Pierre Véran, Lianqi Wang, Kai Wei, Ivan Wevers, "TMT adaptive optics program status report," Proc. SPIE 8447, Adaptive Optics Systems III, 84471J (13 September 2012); doi: 10.1117/12.927046

Event: SPIE Astronomical Telescopes + Instrumentation, 2012, Amsterdam, Netherlands 


\title{
TMT adaptive optics program status report
}

\author{
Brent L. Ellerbroek ${ }^{a}$, Sean M. Adkins ${ }^{b}$, David R. Andersen ${ }^{c}$, Jenny Atwood ${ }^{c}$, \\ Arnaud Bastard ${ }^{d}$, Yong Bo ${ }^{e}$, Marc-Andre Boucher ${ }^{c}$, Corinne Boyer ${ }^{a}$, Peter W. G. Byrnes ${ }^{c}$, \\ Kris Caputa ${ }^{c}$, Shanqiu Chen ${ }^{f}$, Carlos Correia ${ }^{c}$, Raphael Cousty ${ }^{d}$, Joeleff T. Fitzsimmons ${ }^{c}$, Luc \\ Gilles $^{a}$, James Gregory ${ }^{g}$, Glen Herriot ${ }^{c}$, Paul Hickson ${ }^{h}$, Alexis Hill ${ }^{c}$, John Pazder $^{c}$, Hubert \\ Pagès $^{d}$, Thomas Pfrommer ${ }^{h}$, Vladimir A. Reshetov ${ }^{c}$, Scott Roberts ${ }^{c}$, Jean-Christophe \\ Sinquin $^{d}$, Matthias Schoeck ${ }^{a, c}$, Malcolm Smith $^{c}$, Jean-Pierre Véran ${ }^{c}$, Lianqi Wang ${ }^{a}$, Kai Wei ${ }^{f}$, \\ and Ivan Wevers ${ }^{c}$ \\ ${ }^{a}$ TMT Observatory Corp., 1111 S. Arroyo Pkwy, Ste. 200, Pasadena, CA USA 91107; \\ ${ }^{b}$ W. M. Keck Observatory, 65-1120 Mamalahoa Hwy., Kamuela, HI USA 96743; \\ ${ }^{c}$ National Research Council Canada, 5071 W. Saanich Rd., Victoria, BC Canada V9E 2E7; \\ ${ }^{d}$ CILAS, 8 Ave. Buffon - ZI La Source, Orleans, France 45100; \\ e Technical Institute of Physics and Chemistry, 2 Beiyitiao St., \\ Zhongguancun, Haidian District, Beijing, China; \\ Institute of Optics and Electronics, P.O. Box 350, Shuangliu, Chengdu, Sichuan, China; \\ ${ }^{9}$ MIT Lincoln Lab., 244 Wood St., Lexington, MA USA 02420; \\ ${ }^{h}$ The Univ. of British Columbia, 6224 Agricultural Rd., Vancouver, BC Canada V6T $1 Z 1$
}

\begin{abstract}
We provide an update on the development of the first light adaptive optics systems for the Thirty Meter Telescope (TMT) over the past two years. The first light AO facility for TMT consists of the Narrow Field Infra-Red AO System (NFIRAOS) and the associated Laser Guide Star Facility (LGSF). This order $60 \times 60$ laser guide star (LGS) multi-conjugate AO (MCAO) architecture will provide uniform, diffraction-limited performance in the $\mathrm{J}, \mathrm{H}$, and $\mathrm{K}$ bands over 17-30 arc sec diameter fields with 50 per cent sky coverage at the galactic pole, as is required to support TMT science cases. Both NFIRAOS and the LGSF have successfully completed design reviews during the last twelve months. We also report on recent progress in AO component prototyping, control algorithm development, and system performance analysis.
\end{abstract}

Keywords: Adaptive optics programs, extremely large telescopes

\section{INTRODUCTION}

The first light facility AO system for TMT is the Narrow Field Infra-Red AO System (NFIRAOS), which will provide diffraction-limited performance in the $\mathrm{J}, \mathrm{H}$, and $\mathrm{K}$ bands over 17-30 arc sec diameter fields with $50 \%$ sky coverage at the galactic pole. This is accomplished with order $60 x 60$ wavefront sensing and correction, two deformable mirrors (DMs) conjugate to ranges of 0 and $11.2 \mathrm{~km}, 6$ sodium laser guide stars (LGSs) in an asterism with a diameter of 70 arc sec, and three low order (tip/tilt or tip/tilt/focus), infra-red natural guide star (NGS) wavefront sensors (WFSs) deployable within a 2 arc minute diameter patrol field. The first light LGS asterism is generated by the Laser Guide Star Facility (LGSF), which initially incorporates 6 20-25W class laser systems mounted to the telescope elevation journal, a mirror-based beam transfer optics system, and a $0.4 \mathrm{~m}$ diameter laser launch telescope located behind the TMT secondary mirror.

Significant progress has been made in developing the first-light $\mathrm{AO}$ architecture since our previous paper presented in 2010. ${ }^{1}$ The NFIRAOS team has now fully implemented the design changes and simplifications associated with the new distortion-free, dual OAP relay design form, ${ }^{2}$ and have completed their Preliminary Design

Further author information: B.L.E.: E-mail: brente@caltech.edu, Telephone: 16263951620

Adaptive Optics Systems III, edited by Brent L. Ellerbroek, Enrico Marchetti, Jean-Pierre Véran, Proc. of SPIE Vol. 8447, 84471J · C 2012 SPIE · CCC code: 0277-786/12/\$18 · doi: 10.1117/12.927046 
Update Review in December, 2011. Work is continuing on component prototyping for NFIRAOS, componentlevel opto-mechanical designs, performance analysis, and interface definition.

Additionally, the LGSF effort is now the responsibility of the Institute of Optics and Electronics (IOE) in Chengdu, China. Their Conceptual Design Update Review was completed in June, 2011. Work on the LGSF Preliminary Design is expected to commence in mid-2013, following further progress on the design of the telescope structure, more specifically the space envelope allocation for the laser platform and the geometry and dimensions of the secondary mirror support struts.

Protoyping activities continue for laser systems, WFS detectors, and deformable mirrors, as well as the associated detector readout systems and DM drive electronics. Processor benchmarking is also underway for real-time-controller (RTC) design concepts. and AO performance estimates and error budgets have been further detailed. Some of the modeling and analysis topics which have received particular attention include (i) high precision astrometry, (ii) turbulence $\left(C_{n}^{2}\right)$ profile estimation from LGS WFS measurements, (iii) LGS fratricide, (iv) sodium layer range tracking, (v) the derivation of NFIRAOS optical surface specifications from AO performance requirements, (vi) PSF reconstruction, and (vii) further optimization of sky coverage and the peformance of the tip/tilt and low-order NGS (or plate scale) mode control loops. Field tests continue at the University of British Columbia LIDAR facility to measure the spatial and temporal variability of the sodium layer, ${ }^{3}$ and to characterize the sodium coupling efficiency of candidate laser systems for TMT. Finally, the Herzberg Institute of Astrophysics is developing an MCAO testbed for further development and validation of the NFIRAOS control algorithms. ${ }^{4}$

\section{FIRST LIGHT AO REQUIREMENTS}

The principal top-level requirements for the TMT first-light AO system have been discussed previously ${ }^{1}$ and have remained unchanged since the last AO Systems Conference in 2010. They are summarized in Table 1 below. Diffraction-limited image quality in the near IR, with high sky coverage and a corrected field-of-view significantly larger than the anisoplanatic patch size, enables a wide range of observations described in the TMT Science Case. ${ }^{5}$ Taken as a whole, achieving this set of requirements will represent a significant improvement beyond the best performance currently achieved with LGS AO on existing 8-10 meter class telescopes.

Table 1. Top-level first light AO requirements for TMT

\begin{tabular}{ll}
\hline Optical throughput & $85 \%$ in J, H, K, and I bands \\
Thermal emission & $15 \%$ of ambient sky and telescope \\
RMS wavefront error & $187 \mathrm{~nm}$ RMS on axis; 191 nm RMS on 17 " FoV \\
Sky coverage & $50 \%$ for Galactic pole guidestar densities \\
Photometric accuracy & $2 \%$ over 30 " at $\lambda=1 \mu \mathrm{m}$ for a 10 minute exposure \\
Astrometric accuracy & $50 \mu$ arc sec over $30 "$ in H band for a 100 second exposure \\
Output ports & 3 ports, f/15 with a 2' FoV \\
\multicolumn{2}{c}{ Available at first light with low risk and acceptable cost } \\
\hline
\end{tabular}

These requirements already determine many of the basic design choices for our first light AO system, including:

- A cooled (-30C) optical system for low thermal emission, with a strong effort made to minimize the number of surfaces;

- High order $(60 \times 60)$ wavefront compensation at a high update rate $(800 \mathrm{~Hz})$ for the required wavefront quality;

- Multi-conjugate AO (MCAO) with 6 guide stars and two deformable mirrors for the required field-of-view and astrometric/photometric accuracy;

- LGS AO for sky coverage; and

- Tip/tilt and tip/tilt/focus NGS wavefront sensing in the near IR with a 2 arc min patrol field, again for sky coverage. 


\section{DERIVED ARCHITECTURE AND TECHNOLOGY CHOICES}

Fig. 1 illustrates the TMT AO system architecture derived from the above requirements. It includes two principle hardware subsystems: The Laser Guide Star Facility (LGSF) and the Narrow Field Infra-Red AO System (NFIRAOS).*

NFIRAOS is mounted on the telescope Nasmyth platform, and provides output ports for three science instruments. Multi-conjugate wavefront correction is implemented using a pair of piezostack deformable mirrors (DMs), which have been selected as the most technically mature option for the required order of correction with a pupil diameter suitable for a 2 arc minute diameter field. Tip/tilt corrections are applied using a tip/tilt stage, which serves as the mount for the ground-conjugate deformable mirror. This design choice reduces the number of optical surfaces, which is reduced further by performing field derotation via bearings at each instrument interface. Further information on the design of NFIRAOS and the status of AO component development is given in Sections 4.1 and 5 below, and in a related paper at this conference. ${ }^{2}$

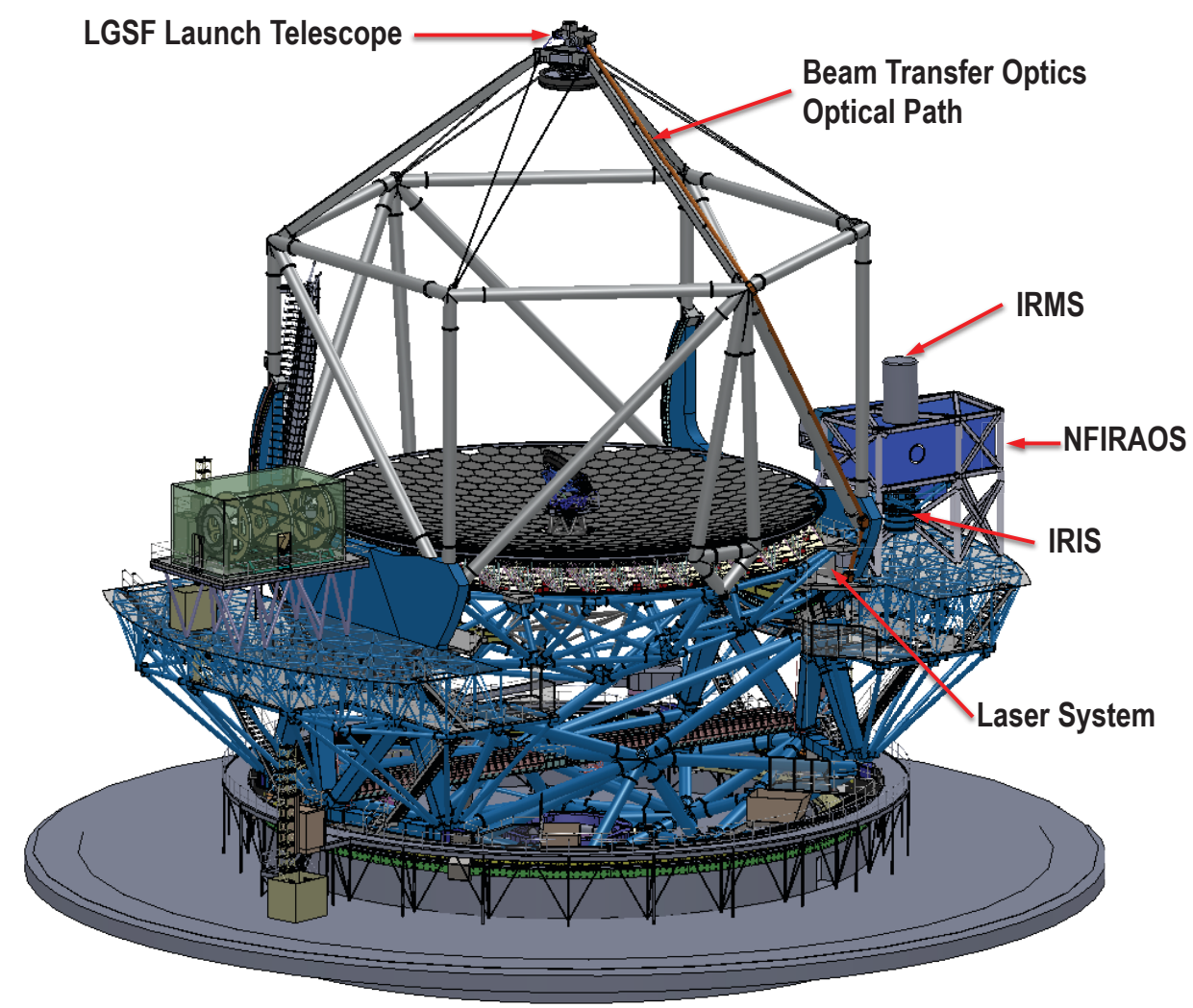

Figure 1. First light AO system architecture for TMT

The LGSF projects 6 sodium LGS in the NFIRAOS asterism, and can also be configured to generate 3 other asterisms for future TMT AO systems. The laser beams are projected from a $0.4 \mathrm{~m}$ launch telescope located behind the TMT secondary mirror; the so-called "center launch" configuration reduces the magnitude of the LGS elongation and consequently the number of pixels needed in the LGS WFS detectors. The LGSF employs solid-state laser technology to generate the six sodium laser guide stars; both sum-frequency Nd:YAG and frequency-doubled Raman fiber laser systems are considered to be practical design options. $20 \mathrm{~W}$ of laser power per guide star, with good coupling efficiency to the mesospheric sodium layer, is required to obtain a sufficient WFS signal level for satisfactory performance.

\footnotetext{
* It additionally includes the AO Executive Software (AOESW) System, not illustrated in the figure.
} 
The lasers themselves are mounted on the telescope elevation journal. This shortens and simplifies the beam path to the laser launch telescope, but requires lasers which are relatively compact, low-maintenance, and operable in a variable gravity orientation. This is feasible thanks to recent advances in demonstrated laser systems. Finally, the beam transport system is implemented using mirrors instead of optical fibers, given the length of the path and the power level of 20 Watts per beam.

\section{SUBSYTEM DESIGN TRADEOFFS AND RECENT PROGRESS 4.1 Narrow Field IR AO System (NFIRAOS)}

The NFIRAOS effort has successfully completed a Preliminary Design Update Review in December of 2011. This "update" is based upon the new optical design form, Fig. 2a, which employs pair of OAP relays to reduce image distortion to a few thousandths of one per cent as necessary for high precision astrometry. Fig. $2 \mathrm{~b}$ illustrates the updated opto-mechanical layout, and Fig. 3 illustrates the NFIRAOS optical enclosure, support structure, and electronics enclosure on the TMT Nasmyth platform.

This iteration of the overall optical design has also led to number of related simplifications and improvements, including:

- The dual OAP relay design form has simplified the design of the LGS WFS optical path and improved performance, as the wavefront aberrations associated with observing a guide star at finite range through the back-to-back relays largely cancel.

- The design of the LGS source simulator optics has been similarly simplified.

- There is now space to include a phase screen turbulence simulator in the system.

- The previous large optical bench has been replaced by a stiffer space frame support system, so that the lowest resonant frequency of the system has not decreased in spite of the increased size.

- A new design approach for the thermal enclosure, using a buried cold plate within the insulation panels, has eliminated the need for specialized materials and reduced cost.

- A review of requirements has also led to additional simplifications in the acquisition sensor (smaller FoV), the NGS "truth" WFS used to detect aberrations caused by variations in the sodium layer profile (now $12 \times 12$ subapertures), and the instrument selection fold mirror (now one-axis instead of two).

Figure 4 is a simplified schematic block diagram of NFIRAOS highlighting these design simplifications.

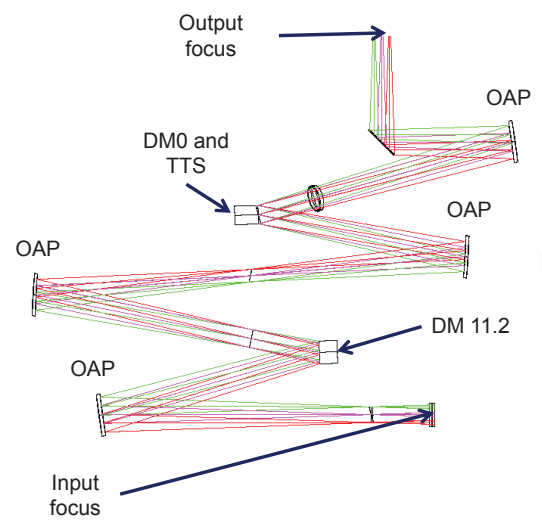

a) 4 off-axis parabola (OAP) optical design

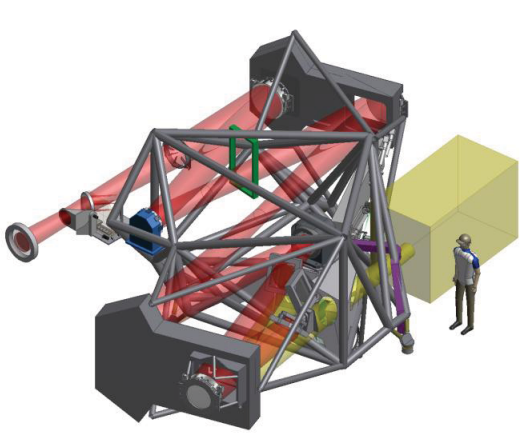

b) Opto-mechanical layout

Figure 2. Revised NFIRAOS optical design and opto-mechanical layout 


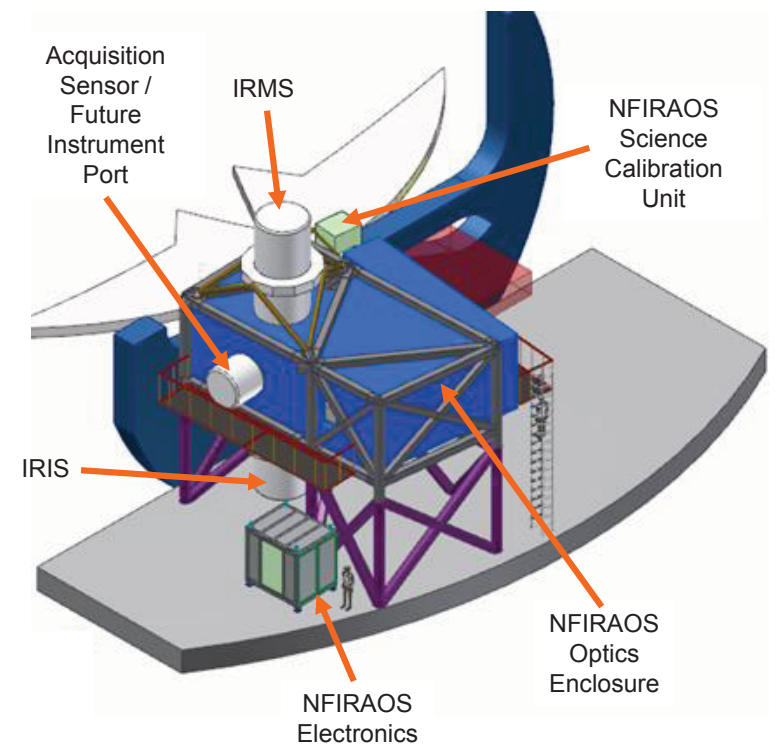

Figure 3. NFIRAOS on the TMT Nasmyth platform

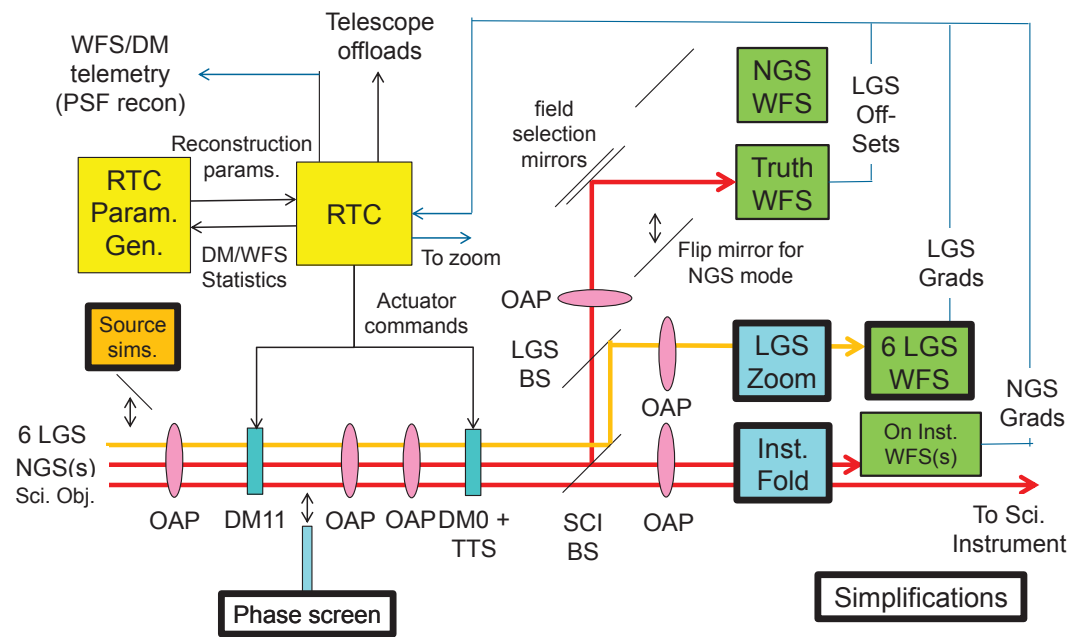

Figure 4. NFIRAOS simplified block diagram

\subsection{Laser Guide Star Facility (LGSF)}

The development of the TMT Laser Guide Star Facility is now the responsibility of the Institute of Optics and Electronics (IOE) in Chengdu, China. ${ }^{\dagger}$ Beginning in late 2010, IOE has updated the Conceptual Design of the LGSF to improve performance and maintain compatibility with evolutionary changes in the designs of the telescope structure and candidate laser systems. The most visible updates to the design include:

- A refined layout for mounting up to 8 guidestar laser systems on a platform attached to the inside of the telescope elevation journal (Fig. 5),

- a new Beam Transfer Optics (BTO) path from the laser location to the telescope top end, which has been updated to match the $60^{\circ}$ rotation of the secondary mirror support structure (Fig. 1 above), and

\footnotetext{
${ }^{\dagger}$ See section 5.4 below for a discussion of the guide star laser system itself.
} 
- an updated and repackaged LGSF top end design (Fig. 6). The height of LGSF top end has been reduced by 0.3 meters, the launch telescope aperture diameter is now 0.4 meters, and an LGS acquisition camera has been included in the design.

IOE has also iterated the designs for the electronics and software subsystems, and updated the budgets for mass, optical performance, and heat dissipation, as part of their conceptual design update. They are now developing cost and schedule estimates for completing the design and construction of the LGSF at IOE.

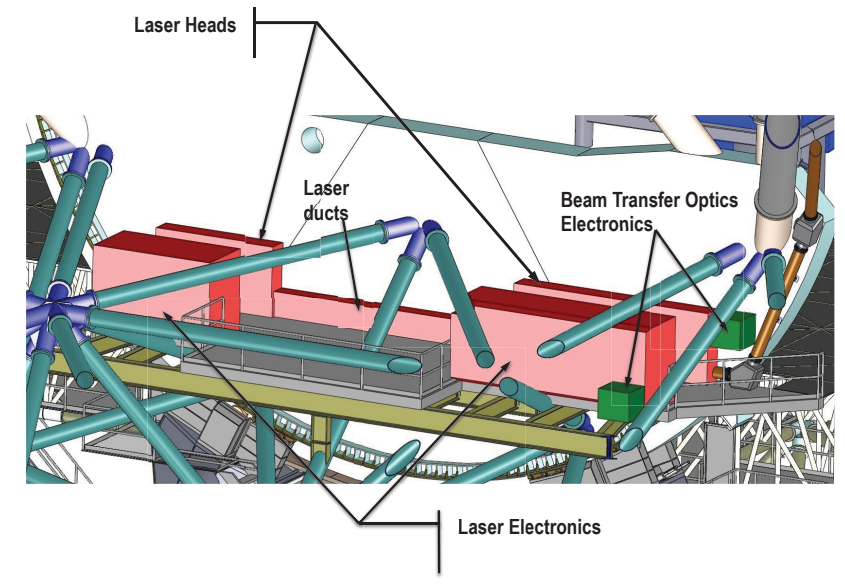

Figure 5. Laser systems (red) mounted on the telescope elevation journal. The orange tube on the right-hand side is the first segment of the beam transfer optics path.

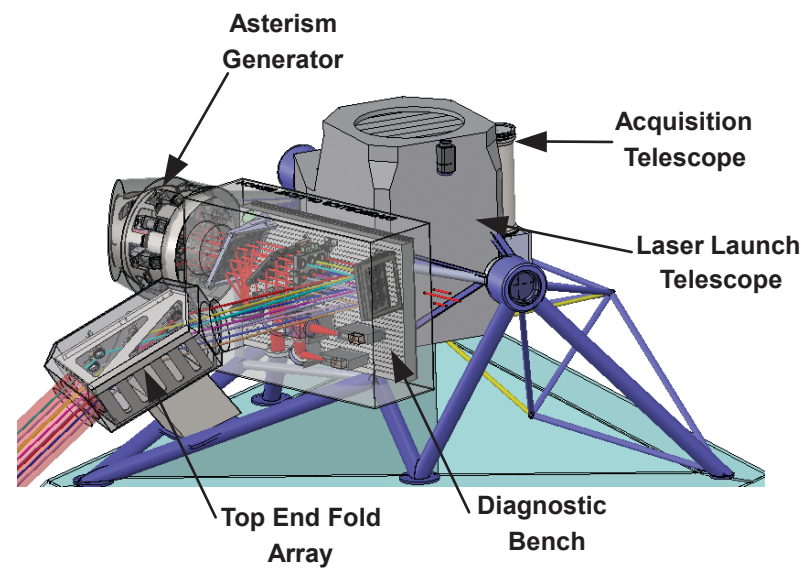

Figure 6. Updated design for the LGSF top end

\section{AO COMPONENT DEVELOPMENT}

Table 2 summarizes the key top-level requirements for the principal components of the first-light AO systems. Design and prototyping progress since our last progress report in 2010 is outlined below for four of these components. See reference [6] in this volume for a discussion of RTC processor benchmarking.

\subsection{Deformable mirrors}

CILAS is now progressing with the final design of the NFIRAOS order $63 \times 63$ and $76 \times 76$ DMs. An order $6 \times 60$ prototype is also in fabrication, which will be tested during the second half of 2012 to validate several 
Table 2. First light AO component requirements summary

\begin{tabular}{ll}
\hline Deformable mirrors & $63 \times 63$ and $76 \times 76$ actuators with $5 \mathrm{~mm}$ spacing \\
& $10 \mu$ m stroke and $5 \%$ hysteresis at $-30 \mathrm{C}$ \\
Tip/tilt stage & $500 \mu$ rad stroke with $0.05 \mu$ rad noise \\
& $80 \mathrm{~Hz}$ bandwidth \\
& $240 \times 240$ pixels, $4 \times 4$ pixels per subaperture \\
NGS WFS detector & 0.8 quantum efficiency, 1 electron noise at $10-800 \mathrm{~Hz}$ \\
& $60 \times 60$ subapertures with $6 \times 6$ to $6 \times 15$ pixels each \\
LGS WFS detectors & 0.9 quantum efficiency, $\sim 3$ electrons noise at $800 \mathrm{~Hz}$ \\
& $1024 \times 1024$ pixels,with subarray readout on $\sim 8 \times 8$ pixel windows \\
IR OIWFS detectors & 0.6 quantum efficiency, $\sim 3$ electrons noise at $10-200 \mathrm{~Hz}$ frame rates \\
& $20 \mathrm{~W}(25 \mathrm{~W}$ without backpumping $), M^{2} \leq 1.17$ \\
Sodium guidestar lasers & Sodium coupling efficiency of 130 photons-m $/ 2 / \mathrm{s} / \mathrm{W} /$ atom \\
& Solve $35 \mathrm{k} \times 7 \mathrm{k}$ reconstruction problem at $800 \mathrm{~Hz}$ \\
\hline
\end{tabular}

important aspects of the DM design and the manufacturing processes. ${ }^{7}$ These include the qualification of a new supplier for the piezo material, experimental verification of FEA models for variations in mirror figure between $20 \mathrm{C}$ and $-30 \mathrm{C}$, confirmation of the stability of the mirror "shape at rest," and accelerated lifetime testing of the actuators and their electrical contacts. Actuator-level tests of (free) stroke and hysteresis indicate that these two requirements should be met with some margin. Fig. 7 illustrates the $6 \times 60$ prototype as of early May 2012, with the facesheet material attached but not yet ground or polished.

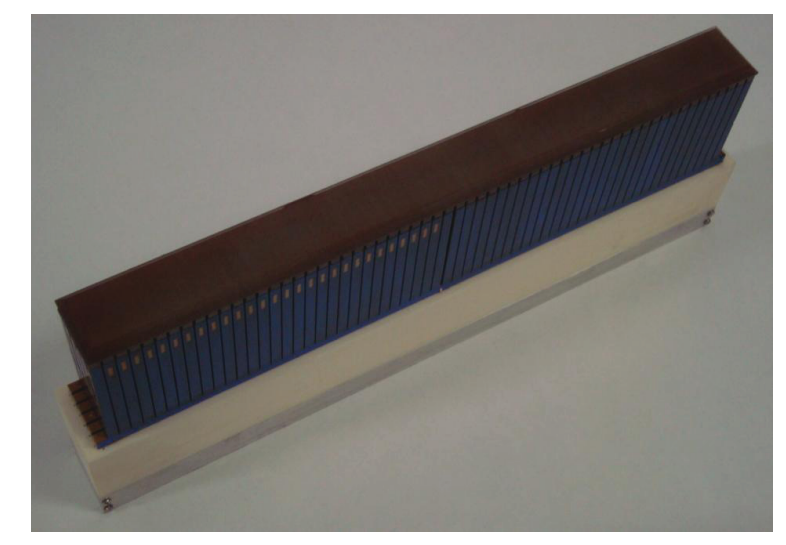

Figure 7. $6 \times 60 \mathrm{DM}$ breadboard with facesheet material attached, but not yet ground or polished (CILAS)

HIA has also developed a reference design for the DM high-voltage drive electronics, and successfully tested a 32 channel prototype board. ${ }^{8}$ The order $6 \times 60$ CILAS prototype will be delivered to HIA later in 2012 for integrated testing with a full scale (96 channel) prototype of the drive electronics.

\subsection{NGS and LGS wavefront sensor detectors}

As described previously, the LGS WFS design for NFIRAOS features a "polar coordinate" CCD array with a pixel geometry customized for use with elongated laser guide star images. Pixel counts and read rates are consequently reduced by roughly a factor of three in comparison with a standard CCD array geometry, and detector read noise is further reduced through the use of the planar JFET amplifier. In 2011, MIT Lincoln Laboratory completed the fabrication, probing, dicing, front-side processing, and packaging of several singlequadrant, $30 \times 30$ subaperture prototypes of this device (see Fig. 8 ). The CCDs produced in this wafer run have demonstrated good yield, correct functional operation, 3.5 noise electrons at the operational pixel read rate, and the expected charge-diffusion OTF between pixels. ${ }^{9}$ 
Additionally, Lincoln Laboratory has now thinned several additional prototype polar coordinate CCDs for back-illuminated quantum efficiency measurements later this year. Several $256 \times 256$ devices, intended for use in the NFIRAOS NGS WFS camera, were processed concurrently and will also be packaged for testing.

Finally, a conceptual design study for the associated camera readout electronics (for both CCDs) was successfully completed in mid-2011 at Keck Observatory. As of this writing, TMT is preparing to release a Request for Information to survey the market for any off-the-shelf detector controllers which may meet our requirements in this area.

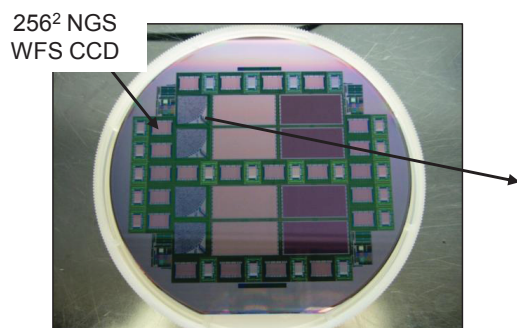

a) Prototype wafer run funded by TMT, Keck and USAF

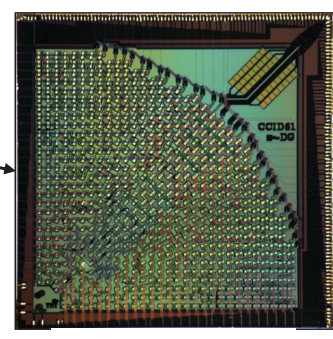

b) Single quadrant prototype polar coordinate CCD

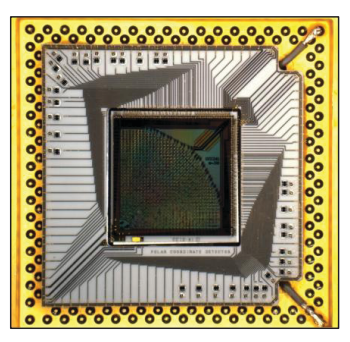

c) Packaged prototype CCD

Figure 8. Fabrication and packaging of the polar coordinate CCD prototype (MIT Lincoln Laboratory)

\subsection{NGS on-instrument WFS detectors}

The Teledyne H2RG detector remains the baseline for use in the on-instrument WFS (OIWFS) cameras for the NFIRAOS science instruments. The detector satisfies the TMT requirements for the number of pixels, quantum efficiency in $\mathrm{J}$ and $\mathrm{H}$ bands, and detector read noise on small subarrays at frame rates of 10 to $200 \mathrm{~Hz}$. We are now considering the costs and sky coverage benefits of extending the OIWFS passband to include K band.

\subsection{Guidestar lasers}

The Technical Institute of Physics and Chemistry (TIPC)in Beijing, China has developed a prototype solid state Nd:YAG, sum frequency laser system for the TMT LGSF. The laser satisfies the fundamental requirements for mass, dimensions, laser power, and beam quality, and was successfully tested on the sky in early 2011 (see Fig. 9). ${ }^{10}$ A new prototype, now under development, will include "repumping" of the sodium D2b line to increase the laser's sodium coupling efficiency and the guidestar photon return. Atomic physics modeling indicates that the laser pulse format will meet the TMT requirement for coupling efficiency when this feature is implemented. ${ }^{11}$ The new prototype will also be tested on the sky later this summer at the University of British Columbia LIDAR facility to confirm these performance predictions.
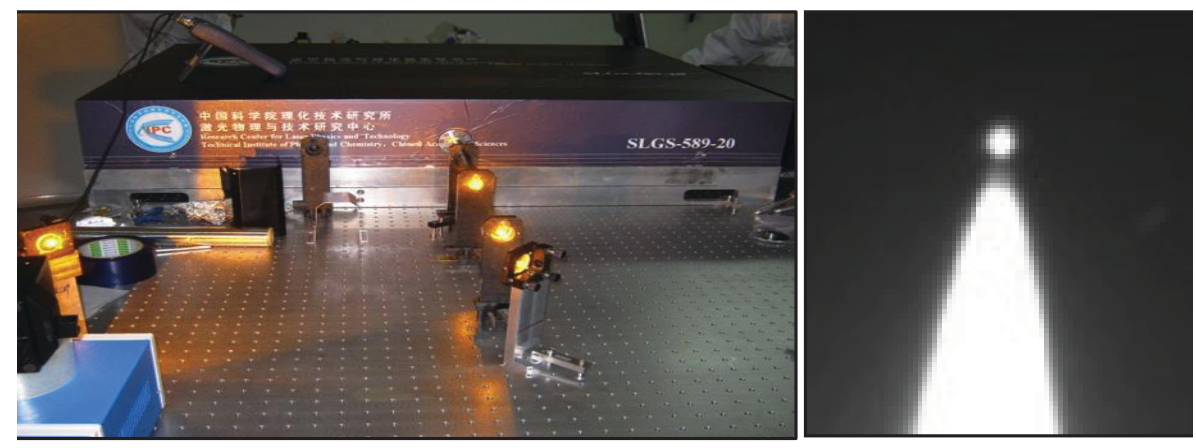

Figure 9. TIPC prototype guidestar laser (left) and on-sky tests in China (right) 


\section{MODELING AND SYSTEM PERFORMANCE ANALYSIS}

Some of the modeling and analysis topics which have received particular attention since the last TMT AO progress report in 2010 include:

- Maintenance of the NFIRAOS error budgets, and evaluation/optimzation of NFIRAOS sky coverage; ${ }^{12,13}$

- sodium coupling efficiency for the candidate guidestar laser systems; ${ }^{11}$

- the impact of transient meteor trails on LGS WFS performance; ${ }^{14}$

- atmospheric $C_{n}^{2}(h)$ profile estimation using LGS WFS telemetry and a SLODAR algorithm; ${ }^{15}$

- investigation of innovative real-time estimation and control algorithms, including (i) LQG control for improved error rejection of the low-order wavefront error modes, specifically vibration suppression, ${ }^{16}$ (ii) "minimual variance split tomography," a computationally efficient implementation of a minimal variance estimator for optimally blending the LGS and low-order NGS WFS measurements, ${ }^{17}$ and (iii) "distributed," or approximate, Kalman filtering for higher-order wavefront control in NFIRAOS;

- PSF reconstruction; ${ }^{18}$ and finally

- a variety of ongoing studies, motivated by the NFIRAOS design review last fall, to assess and optimize high contrast imaging with NFIRAOS, ${ }^{19}$ develop an error budget for high precision astrometry, and review the choice of the OIWFS spectral passband to optimize NFIRAOS sky coverage.

Further information on some of these topics is summarized below and in the references given above, including several other papers at this conference.

\subsection{Wavefront Error and Sky Coverage}

The top-level estimate for the NFIRAOS delivered wavefront error (WFE) has remained relatively stable since our last progress report, as summarized in Table 3 below. The modeling effort behind such estimates has benefited significantly from porting our simulation code, MAOS, to GPU processors, which can now simulate the NFIRAOS AO control loop with a 100:1 ratio between wallclock time and NFIRAOS time. ${ }^{6} 100$ seconds of NFIRAOS time can be simulated in less than 3 hours, for example. This level of computational efficiency enables extensive studies of sky coverage and low-bandwidth background processes that would not be possible otherwise.

For example, Fig. 10 illustrates the sky coverage achieved by NFIRAOS over the full sky under median atmospheric conditions, expressed as the probability of achieving a delivered RMS WFE of $191 \mathrm{~nm}$ or less at culmination. Sky coverage at the north Galactic pole is about 70 percent; good to excellent sky coverage is achieved for all targets culminating above a zenith angle of about 45 degees, and even lower angles near the plane of the galaxy.

\subsection{MCAO and Precision Astrometry/Photometry}

The NFIRAOS requirements for precision astrometry and photometry are now receiving considerable attention. In comparison with other $\mathrm{AO}$ modes, $\mathrm{MCAO}$ can improve astrometry and photometry on fields significantly larger than the isoplanatic angle by providing diffraction-limited resolution with a uniform, stable PSF. For example, Fig. 11 below compares the performance of NFIRAOS against a laser tomography AO (LTAO) system with identical AO components but only a single ground-conjugate deformable mirror. The difference in PSF uniformity is readily apparent, even for $\mathrm{K}$ band observations in the 17" field-of-view for the IRIS imager.

PSF uniformity on a larger corrected field improves photometric and astrometric accuracy in multiple ways. For the AO system and atmospheric parameters considered in Fig. 11, for example, a 10 per cent uncertainty in seeing results in less than a 1 per cent peak error in relative photometry using NFIRAOS on a 30" field of view, since the variation in AO performance is nearly field-independent. The corresponding photometry error for LTAO is about 15 per cent. Turning to astrometry, MCAO improves performance in at least 4 separate ways: 
Table 3. NFIRAOS RMS WFE performance summary at zenith, with median seeing and galactic pole guidestar densities.

\begin{tabular}{lc}
\hline Description & RMS WFE, nm \\
\hline Delivered performance & 191 \\
Higher order wavefront error (LGS controlled) & 178 \\
First order turbulence compensation & 135 \\
Best fit wavefront correction error & 96 \\
Noise free wavefront estimation error & 78 \\
Servo lag & 21 \\
WFS noise and nonlinearity & 50 \\
Opto-mechanical implementation errors & 79 \\
TMT pupil misregistration & \multicolumn{2}{c}{12} \\
Telescope/enclosure wavefront & \multicolumn{2}{c}{49} \\
NFIRAOS wavefront & 53 \\
Science instrument wavefront & 30 \\
AO components and higher-order effects & 85 \\
Deformable mirrors & \multicolumn{2}{c}{51} \\
LGS WFS and sodium layer & \multicolumn{2}{c}{48} \\
Control algorithm & 51 \\
Tip/tilt and plate scale (NGS controlled) & 17 \\
Telescope windshake & 20 \\
Telescope vibration and tracking error & 44 \\
Turbulence and WFS measurement noise & 47 \\
Contingency & \\
\hline
\end{tabular}

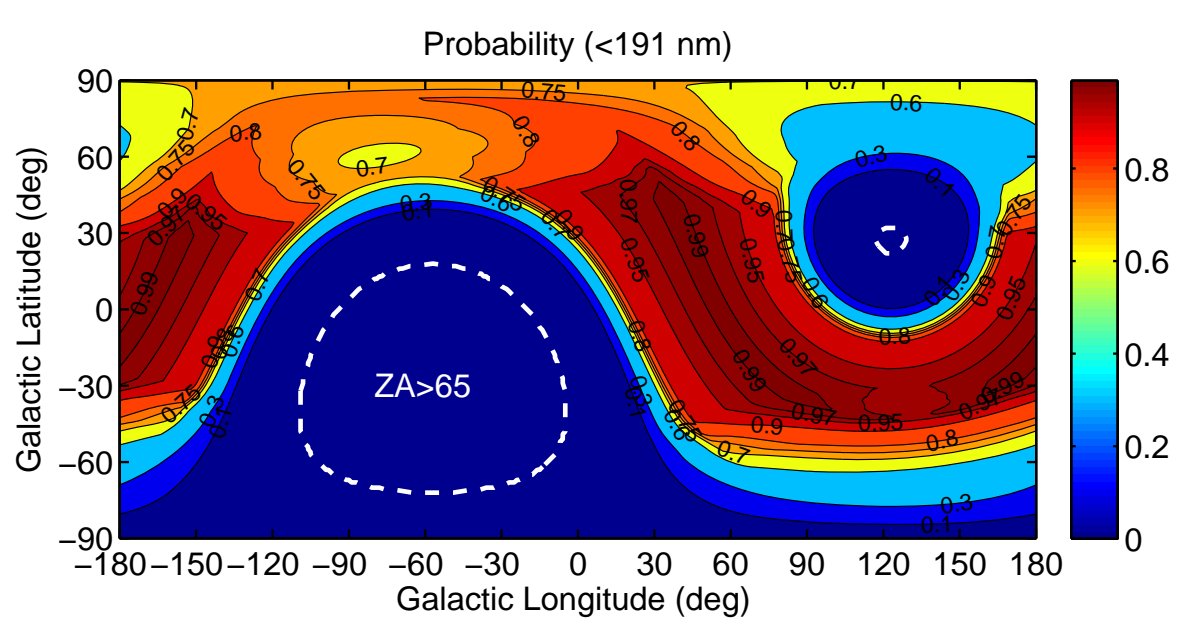

Figure 10. NFIRAOS sky coverage, expressed as the probability of achieving $\leq 191 \mathrm{~nm}$ RMS WFE at culmination with median seeing. The dashed lines indicate targets which culminate below the TMT limit of 65 degrees.

- It provides real-time correction of atmospheric turbulence modes which distort the image;

- it significantly reduces astrometric errors due to residual atmospheric "speckles," even for time-averaged images. For the same scenario considered in Fig. 11, for example, the astrometric errors due to atmospheric speckle in a 15-second NFIRAOS image range from 9-13 micro arc seconds in K, H, and J bands. For LTAO, the corresponding errors are in the range of 40 to 190 micro arc seconds.

- MCAO increases the corrected field-of-view available for imaging reference stars with known coordinates, as needed to calibrate systematic opto-mechanical sources of image distortion; and finally,

- as illustrated on the left-hand side of Fig. 11, a uniform PSF with a stable diffraction limited core reduces confusion and the contamination of astrometric measurements by nearby bright stars. 


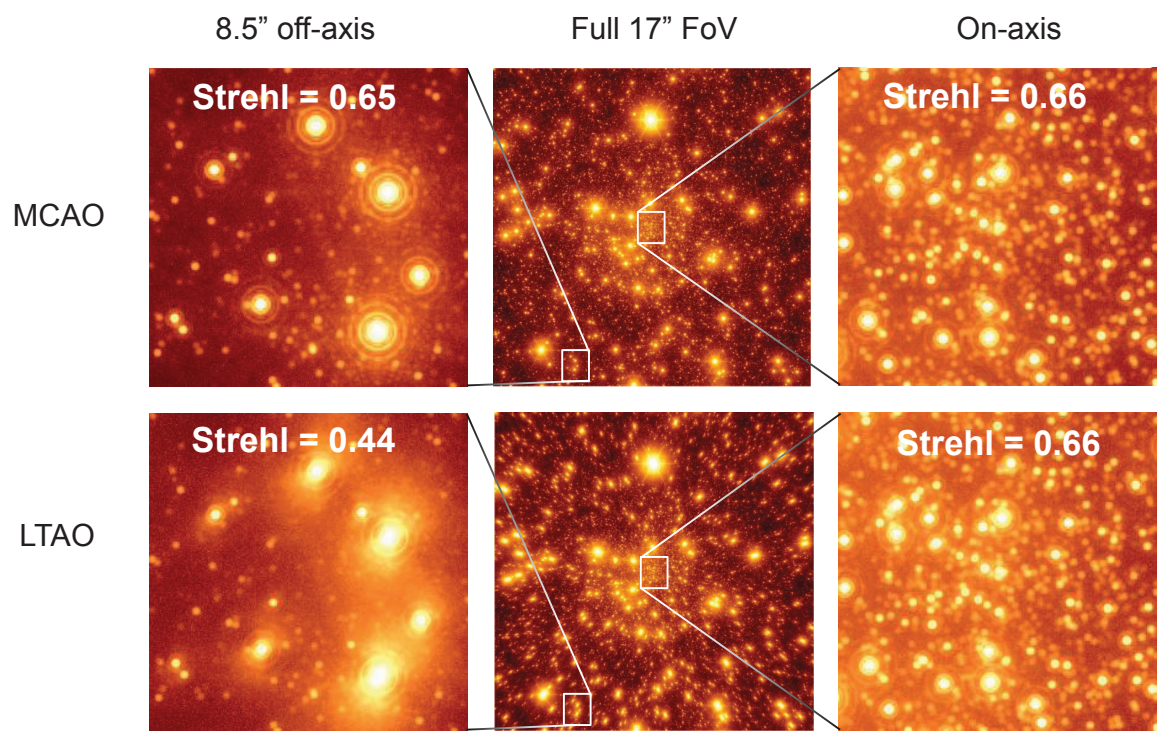

Figure 11. Simulated NFIRAOS (top) and LTAO (bottom) K band image of the Galactic Center with a 17" square field of view (Leo Myer, UCLA).

In other words, the degree of atmospheric turbulence compensation provided by MCAO in NFIRAOS is fundamental to achieving the TMT requirements for precision astrometry and photometry as given in Table 1 above. Although it is true that quasi-static DM calibration errors are an additional source of astrometry error for MCAO not found in other AO modes, a recently developed Fourier domain model for this effect indicates a peak sensitivity of about 0.15 micro arc second of RMS image distortion on a 17 arc sec field per nanometer of wavefront calibration error for the NFIRAOS parameters. Feasible levels of DM calibration accuracy will be sufficient for TMT requirements.

\section{SUMMARY}

Significant progress has continued in developing the first light AO architecture for TMT since our last progress report in 2010. The preliminary design of NFIRAOS has been successfuly completed, including the implementation of the new 4-OAP, distortion-free optical design form. IOE has completed a conceptual design update for the Laser Guide Star Facility. Progress in AO component development has also continued, particulary in the areas of deformable mirrors, visible and near IR wavefront sensing detectors, the associated actuator driver and detector readout electronics, and guidestar laser systems. Increasingly high fidelity modeling capabilities predict that performance requirements will be met in term of wavefront quality, sky coverage, and high precision astrometry. We now look forward to the completion of the design and prototyping activities, and the commencement of the TMT construction phase.

\section{ACKNOWLEDGMENTS}

The TMT Project gratefully acknowledges the support of the TMT collaborating institutions. They are the Association of Canadian Universities for Research in Astronomy (ACURA), the California Institute of Technology, the University of California, the National Astronomical Observatory of Japan, the National Astronomical Observatories of China and their consortium partners, and the Department of Science and Technology of India and their supported institutes. This work was supported as well by the Gordon and Betty Moore Foundation, the Canada Foundation for Innovation, the Ontario Ministry of Research and Innovation, the National Research Council of Canada, the Natural Sciences and Engineering Research Council of Canada, the British Columbia Knowledge Development Fund, the Association of Universities for Research in Astronomy (AURA) and the U.S. National Science Foundation. 


\section{REFERENCES}

[1] Ellerbroek, B. et al., "First light adaptive optics systems and components for the Thirty Meter Telescope," in [Adaptive Optics Systems II], Ellerbroek, B., Hart, M., Hubin, N., and Wizinowich, P., eds., Proc. SPIE 7736, (2010).

[2] Herriot, G. et al., "TMT NFIRAOS: adaptive optics system for the Thirty Meter Telescope," in [Adaptive Optics Systems III], Ellerbroek, B., Marchetti, E., and Veran, J.-P., eds., Proc. SPIE 8447, (2012).

[3] Pfrommer, T. and Hickson, P., "Mesopsheric sodium structure variability and its impact on adaptive optics," in [Adaptive Optics Systems III], Ellerbroek, B., Marchetti, E., and Veran, J.-P., eds., Proc. SPIE 8447, (2012).

[4] Veran, J. P. et al., "The HIA MCAO laboratory bench," in [Adaptive Optics Systems III], Ellerbroek, B., Marchetti, E., and Veran, J.-P., eds., Proc. SPIE 8447, (2012).

[5] Thirty Meter Telescope Detailed Science Case, http://www.tmt.org/foundation-docs/TMT-DSC-2007R1.pdf (2007).

[6] Wang, L. and Ellerbroek, B., "Computer simulations and real-time control of ELT AO systems using graphical processing units," in [Adaptive Optics Systems III], Ellerbroek, B., Marchetti, E., and Veran, J.-P., eds., Proc. SPIE 8447, (2012).

[7] Sinquin, J. C. et al., "TMT DMs final design and advanced prototyping results at Cilas," in [Adaptive Optics Systems III], Ellerbroek, B., Marchetti, E., and Veran, J.-P., eds., Proc. SPIE 8447, (2012).

[8] Caputa, K., Herriot, G., Niebergal, J., and Zielinski, A., "Reference design of deformable mirror electronics for ELT AO systems," in [Adaptive Optics Systems III], Ellerbroek, B., Marchetti, E., and Veran, J.-P., eds., Proc. SPIE 8447, (2012).

[9] Adkins, S., "Measured performance of the prototype polar coordinate CCD array," in [Adaptive Optics Systems III], Ellerbroek, B., Marchetti, E., and Veran, J.-P., eds., Proc. SPIE 8447, (2012).

[10] Wei, K., "Photon returns test of the pulsed sodium guide star laser on the 1.8 meter telescope," in [Adaptive Optics Systems III], Ellerbroek, B., Marchetti, E., and Veran, J.-P., eds., Proc. SPIE 8447, (2012).

[11] S. Rochester et al., J. Opt. Soc. Am B, accepted for publication (2012).

[12] Andersen, D. R., Wang, L., Ellerbroek, B., and Herriot, G., "Predicted sky coverage for the TMT MCAO system NFIRAOS," in [AO for ELT 2], Veran, J.-P., Fusco, T., and Clenet, Y., eds., (2012).

[13] Veran, J. P. and Pazder, J., "Tolerancing the fabrication errors of static optical elements for ELT-size widefield AO systems," in [Adaptive Optics Systems III], Ellerbroek, B., Marchetti, E., and Veran, J.-P., eds., Proc. SPIE 8447, (2012).

[14] Herriot, G. and Pazder, J., "Mitigation of transient meteor events in sodium layer by TMT NFIRAOS," in [Adaptive Optics Systems III], Ellerbroek, B., Marchetti, E., and Veran, J.-P., eds., Proc. SPIE 8447, (2012).

[15] Gilles, L. and Ellerbroek, B., "Real-time turbulence profiling with a pair of laser guide star ShackHartmann wavefront sensors for wide-field adaptive optics systems on large to extremely large telescopes," J. Opt. Soc. Am. A 27, 76-83 (2010).

[16] Correia, C. et al., "Optimal control of plate-scale modes in laser-guide-star-based multi-conjuate adaptive optics," in [Adaptive Optics Systems III], Ellerbroek, B., Marchetti, E., and Veran, J.-P., eds., Proc. SPIE 8447, (2012).

[17] Wang, L., Gilles, L., and Ellerbroek, B., "Analysis of the improvement in sky coverage for multiconjugate adaptive optics systems obtained using minimum variance split tomography," Appl. Opt. 50, 3000-3010 (2011).

[18] Gilles, L. et al., "Long exposure point spread function estimation for laser guide star multi-conjugate adaptive optics," to be submitted.

[19] Marois, C., Veran, J. P., and Correia, C., "A Fresnel propagation modeling of NFIRAOS/IRIS high-contrast imaging capabilties," in [Adaptive Optics Systems III], Ellerbroek, B., Marchetti, E., and Veran, J.-P., eds., Proc. SPIE 8447, (2012). 